\title{
Fabrication of a Cylindrical Display by Patterned Assembly
}

\author{
Heiko O. Jacobs,* Andrea R. Tao, Alexander Schwartz, \\ David H. Gracias, George M. Whitesides $\dagger$
}

\begin{abstract}
We demonstrate the patterned assembly of integrated semiconductor devices onto planar, flexible, and curved substrates on the basis of capillary interactions involving liquid solder. The substrates presented patterned, solder-coated areas that acted both as receptors for the components of the device during its assembly and as electrical connections during its operation. The components were suspended in water and agitated gently. Minimization of the free energy of the solder-water interface provided the driving force for the assembly. One hundred and thirteen GaAlAs light-emitting diodes with a chip size of 280 micrometers were fabricated into a prototype cylindrical display. It was also possible to assemble 1500 silicon cubes, on an area of 5 square centimeters, in less than 3 minutes, with a defect rate of $\sim 2 \%$.
\end{abstract}

The assembly of individual devices into integrated systems is a key process in microelectronics and optics. The past three decades have produced a range of new manufacturing technologies focused on assembly: serial pick-and-place, serial wire-bonding, serial packaging, and parallel wafer-to-wafer transfer (1). Each has limitations: Pick-and-place is inefficient with large numbers of components and with components with dimensions of $<100 \mu \mathrm{m}$ because adhesive forces often dominate gravitational forces (2). Both micromanipulator-based assembly and waferto-wafer transfer methods work poorly on nonplanar surfaces, in cavities, and in fabrication of three-dimensional (3D) systems. Serial processes, in general, are slow.

We and others have begun to examine patterned assembly and self-assembly as strategies for fabrication of devices made up of small components (3-9). These assembly methods are intrinsically parallel and have the potential for submicrometer accuracy in positioning. They are relatively insensitive to certain types of errors in registration. Selfassembly has been studied extensively in chemistry and biology, and molecular selfassembly offers a rich menu of examples (10, 11) to use in designing processes that allow fabrication with components larger than molecules $(12,13)$.

Previous demonstrations of patterned assembly to generate functional electrical devices include shape-directed fluidic methods that position electronic devices on plastic

Department of Chemistry and Chemical Biology, Harvard University, 12 Oxford Street, Cambridge, MA 02138, USA.

*Present Address: Department of Electrical Engineering, University of Minnesota, 200 Union Street SE, Minneapolis, MN 55455, USA. E-mail: hjacobs@ ece.umn.edu

†To whom correspondence should be addressed. Email: gwhitesides@gmwgroup.harvard.edu supports $(14,15)$, coplanar integration of segmented integrated circuit (IC) devices into 2D "super chips" by using capillary forces $(16,17)$, solder-based assembly that uses the surface tension between molten solder drops to fabricate 3D electrical networks (18), ring oscillators, and shift-registers (19). Processes based on the surface tension between solder drops and metallic surfaces have been used previously to assemble electronic and mechanical structures; examples include "flipchip" technology (20) and the rotation of parts of microstructures into nonplanar orientations $(21,22)$.

Here we focus on solder-based assembly to integrate device segments onto nonplanar substrates. The dimensions $(\sim 300 \mu \mathrm{m})$ of these components are 30 times smaller than those of previous solder-based self-assemblies $(18,19)$ and are about the same as those assembled by shape-directed fluidic procedures $(3,14,15)$. Our strategy involves use of a substrate that carries solder-coated areas of different size and shape. These areas act as receptors for the units during the assembly (23) and as electrical interconnects. We have demonstrated both the fabrication of an electrically functional cylindrical display containing 113 light-emitting diodes (LEDs) (as a first prototype of a cylindrical display) and the generation of a nonplanar array containing $\sim 1600$ small silicon cubes (as surrogates of microelectronic devices). In each example, the assembly process was completed in less than $3 \mathrm{~min}$.

The experimental strategy for the assembly of components onto planar and nonplanar substrates is shown in Fig. 1. To construct a system that required electrical connectivity of the sort relevant to displays, we used LEDs - unpackaged $\mathrm{GaAs} / \mathrm{GaAlAs}$ LEDs with a chip size of $280 \mu \mathrm{m}$ by $280 \mu \mathrm{m}$ by $200 \mu \mathrm{m}$ - as components. The chips had two contacts: a small circular cathode on the front, and a large square anode covering the back (Fig. 1A). To obtain the large number of chips required, we used a dicing saw to cut a wafer of photodiode material with gold contacts (GaAlAs; \#P3031, Op-
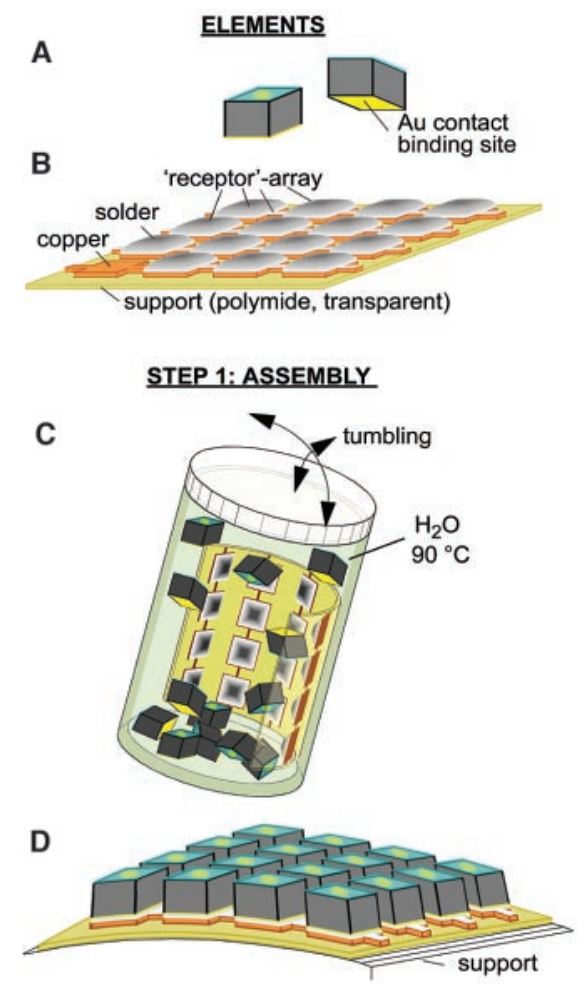

E STEP 2: ALIGNMENT
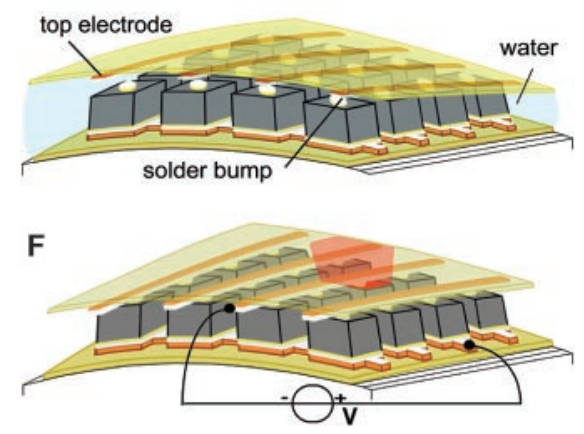

Fig. 1. Procedure used to assemble a functional cylindrical display. (A) Top and bottom views of a LED segment that has two contacts: a small circular Au contact (cathode) on the front, and a large square $\mathrm{Au}$ contact (anode) on the back. (B) Array of solder-coated copper squares supported on a flexible substrate; these squares are the same size as the anodes of the LEDs and act as receptors for the LEDs during the self-assembly. (C) The components are tumbled in a vial at a temperature above the melting point of the solder. (D) Two-dimensional array of the assembled LEDs. (E) Alignment of a top electrode: The copper wires of the top electrode and the cathodic contacts on the front of the LEDs were first dip-coated with solder. The array of wires is prealigned with the array of cathodic solder bumps. At a temperature above the melting point, electrical connections form and the structure self-aligns. (F) Test of the self-assembled display-prototype. 
todiode Corporation, Newbury Park, California) into $280-\mu \mathrm{m}$-wide segments.

To induce the LEDs to assemble into a well-defined array, we fabricated $280-\mu \mathrm{m}$ wide copper squares on a substrate that we coated with a low-melting point (mp) solder $\left(\mathrm{mp} \sim 50^{\circ} \mathrm{C}\right.$; Small Parts, Miami Lakes, Florida, Y-LMA-117) (24). The surface of the liquid solder wets and adheres to the goldcoated contacts on the back side of the LEDs; minimization of the free surface area of the liquid solder drives the assembly. The solder also provides the electrical connection required to operate the device and the mechanical bond required to hold the assembly together. The pattern used in the prototype incorporated 113 receptor sites (eight columns of eight receptors interleaved with seven columns of seven receptors). The procedure to fabricate the patterned solder drops is described in the supplemental material (24).

The prototype display element was fabricated in two steps: (i) the self-assembly of LEDs on the bottom electrode and (ii) the self-alignment of the contacts with the top electrode. Both steps were performed in water in order to reduce capillary and gravitational forces. The water was made slightly acidic $(\mathrm{pH}=3.0)$ with acetic acid to remove metal oxide from the surface of the solder drops (24); an oxide layer-if sufficiently thick-blocked fusion of solder drops and the wetting of metal surfaces.

For the assembly of the LEDs, we inserted the bottom electrode into a cylindrical 1-ml vial with an inside diameter of $5 \mathrm{~mm}$, and added water and LEDs (Fig. 1C). We placed the vial in front of a heat gun and heated the water to a temperature of $90^{\circ} \mathrm{C}$; at this temperature, the solder was molten. We then agitated the components by shaking the vial manually until all receptors had captured a LED (1 to $2 \mathrm{~min}$ ).

The second step of the fabrication sequence was the self-alignment of the top electrode. To prepare for this step, we wetted the gold contacts exposed on top of the LEDs with solder by dipping the assembly into the liquid solder bath. The top electrode was fabricated with the same flexible copperpolyimide composite that was used to fabricate the bottom electrode; this film is transparent and therefore suitable for the fabrication of displays. The film-supported copper wires were coated with low-melting point solder. The center-to-center distance between the wires was the same as the center-to-center distance between diodes. During this step, we positioned the flexible top electrode by hand in roughly the correct position to make contacts with the top surfaces of the LEDs. When the entire structure was heated above the melting point of the solder, the top electrode fused with the solder-coated cathodes of the LEDs, and the entire system adjusted the

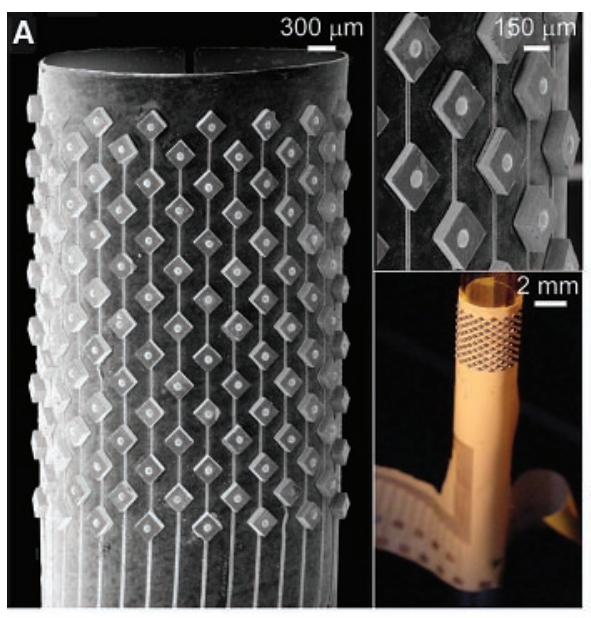

Fig. 2. Scanning electron micrographs (SEMs) of a cylindrical display fabricated in two selfdriven fabrication steps: Patterned assembly of LEDs onto a bottom electrode and self-alignment of a top electrode. (A) SEM and optical images of LEDs that assembled into a regular array. (B) Photograph of the display after selfalignment of the top electrode. (C to E) Photographs of the operating display after the alignment of the top electrode. The display contains 113 LEDs that are assembled in an interleaved fully addressable array with eight
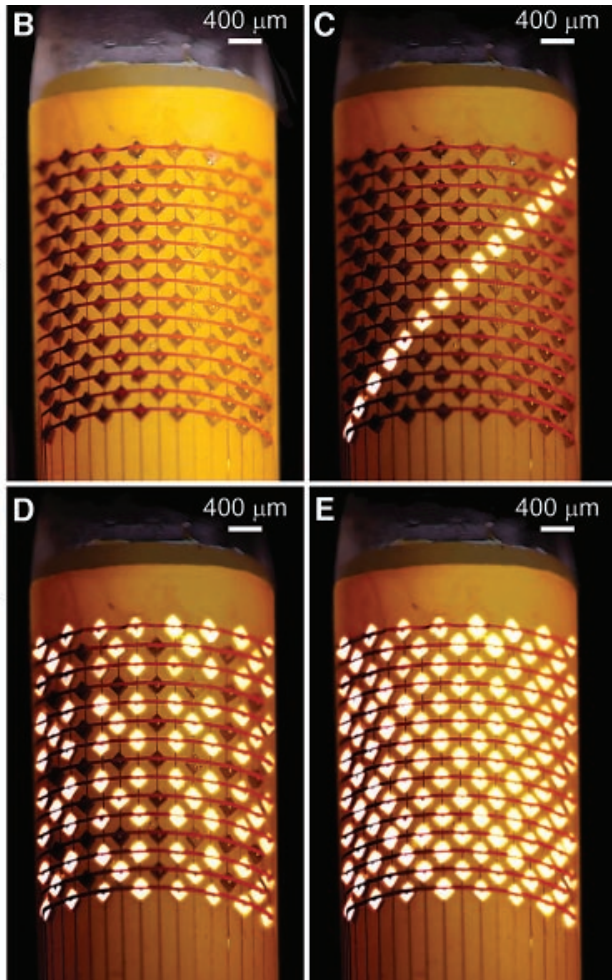

columns of eight receptors interleaved with seven columns of seven on the bottom electrode that connect to 15 rows of crossing copper wires on the top electrode.

position of the components to minimize the overall interfacial energy. This process formed an electrical contact with each LED.

All surfaces (i.e., the copper squares on the bottom electrode, the gold contacts exposed on top of the LEDs, and the copper wires on the top electrode) were dip-coated with the same low-melting point $\left(\mathrm{mp} 50^{\circ} \mathrm{C}\right)$ solder. We designed the contact areas such that the assembled LEDs would stay on their receptors during the dipping process; the surface area of the receptor contact (the area is roughly proportional to the force of adhesion) is eight times larger than the surface area of the top contact.

Figure 2 illustrates the experimental realization of a fully addressable cylindrical display. The assemblies were accomplished by repetitions of two steps, each having a different intensity of agitation. In the first step, we agitated the pieces manually by gently shaking the vial. Using manual agitation allowed us to observe the process and to control the mixing within the system. The progression was not linear with time: It took longer for the first components to settle onto receptors than it did for later ones. We hypothesize that once some segments had assembled, they slowed others tumbling along the surface in their vicinity and allowed them to assemble more rapidly onto nearby receptors. In the second step, we transferred the film with attached LEDs into a vial containing no additional LEDs and agitated the system more vigorously by tapping the vial with a metal rod at a temperature above the melting point of the solder. This step did not affect correctly assembled components, but it did remove two important types of defects - single LEDs assembled on the connecting wire between two adjacent receptors, and two LEDs assembled on a single receptor. The assemblies shown in Fig. 2 were realized by repeating this two-step sequence - attachment of LEDs under gentle agitation and removal of defects with sharper agitation-twice. The entire process - that is, two cycles of attachment of components and removal of incorrectly attached components - took less than $4 \mathrm{~min}$. It was reproduced six times; all assemblies were free of defects. The solder connects the anodes of the diodes electrically to 15 copper wires that form the columns of the display.

The addition of the top electrode (Fig. 2B) required greater precision. We reproduced the patterned alignment of the top electrode several times and found that variations in the height of up to $\sim 70 \mu \mathrm{m}$, and a lateral misfit between the cathodes on the LEDs and the top electrode of up to $\sim 60 \mu \mathrm{m}$, were tolerated; beyond those limits, the final structure had defects. Deviations from the permissible range of sizes and positions resulted from variation in the size of the LEDs $[\sim 30 \mu \mathrm{m}(24)]$ and from inaccuracies in manually positioning the top electrode. Small deviations are compensated by the reflow of solder, the repositioning of LEDs, and the flexibility of the top electrode. 
REP ORT S

Fig. 3. Large area array of silicon segments on flexible nonplanar supports. The array consist of $\sim 1560$ device segments that assembled in less then 3 min onto the solder-based receptors.

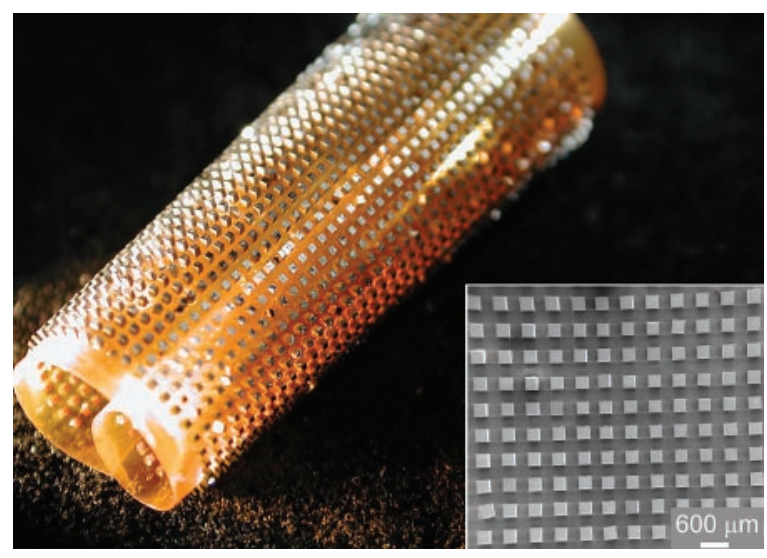

To test the functionality of the LEDs and interconnects, we applied a potential of $2 \mathrm{~V}$ between the top and substrate electrodes. In addition to the cylindrical display shown in Fig. 2, we successfully fabricated planar displays; alignment of the top electrode on planar surfaces generally proceeded with fewer defects than on nonplanar surfaces.

To test the assembly of larger numbers of more readily available components, we used a large $\left(5 \mathrm{~cm}^{2}\right)$, solder-coated pattern on a polyimide foil presenting 1600 (40 by 40 ) receptors, and silicon blocks ( $300 \mu \mathrm{m}$ by 300 $\mu \mathrm{m}$ by $400 \mu \mathrm{m}$ ) with a gold contact on one side (Fig. 3). The procedure used was similar to that in the first assembly step described with the LEDs. The resulting assemblies (in two trials) had 1562 and 1566 silicon segments correctly assembled onto solder-coated copper areas. In both cases, self-assembly achieved $98 \%$ coverage of the patterned solder drops. With $\sim 5000$ components inside the vials, the assembly took about $90 \mathrm{~s}$ to reach steady state and was completed in less than $3 \mathrm{~min}$. The precision in the positioning of the silicon components was $\sim 15 \mu \mathrm{m}$; its angular precision was $\sim 3^{\circ}$. This precision was limited by the non-uniformity of the components (which may have differences in size of $\sim 20 \mu \mathrm{m}$ ) rather than by the assembly process itself.

Receptor design and agitation were crucial parameters that influenced the types and densities of defects. We found three types of defects: (i) single-device segments that assembled by overlapping two adjacent receptors; (ii) single-device segments that assembled onto connecting wires between adjacent receptors; and (iii) two-device segments that assembled onto a single receptor. We were able to remove these defects by increasing the space between adjacent receptors, reducing the width of the connecting wires between adjacent receptors, and changing the levels of agitation during the self-assembly process. We have established neither the minimum size of components that can be assembled without defects, nor the density of defects as a function of time

The remaining defects observed in the large area assemblies ( $\sim 2 \%$ ) (Fig. 3) consisted of missing or misaligned components. These errors were not inherent to the assembly process itself, but were caused by imperfections in both the receptors and the device segments: receptors that were round rather than square, receptors that were only partially coated with solder, receptors that were attached to fragments of components [these fragments originate in the dicing process (24)], receptors where the solder had dissolved (24), and components with partially detached metal contacts. To fabricate the defect-free cylindrical display, a reduction of these remaining defects was a crucial step. We achieved this reduction by careful visual inspection to select solder-coated receptor arrays that had no defects, and by removing fragments of components from the suspension of components using a $200-\mu \mathrm{m}$ mesh filter.

We have demonstrated patterned assembly of $300-\mu \mathrm{m}$-scale components and formation of functional electrical contacts by capillary-driven assembly on both planar and nonplanar surfaces. Solder-based assembly provides a technique for placing small components on surfaces, and of connecting them electrically, where the solder both provides the electrical connectivity and mechanical strength, and drives the process of assembly.

Our initial survey of the errors identified at least some of the parameters that must be controlled to allow consistent assembly with minimal defects. These parameters include the agitation, the design of the solder dots and the receptors, the production of the components, the procedures for removing incorrectly assembled components, and the control of interfacial chemistries. This survey shows no defects that are integral to the process and that intrinsically limit the level of perfection know the intrinsic level of defects.

The prototype display segment that we have used to illustrate patterned assembly is a relatively simple device, with a single type of component and a simple repetitive structure. that can be achieved. We do not currently
Many devices are more complex and contain several different types of components. For such complex systems, we believe that patterned assembly, in combination with other concepts such as shape-selective recognition $(25,26)$ and hierarchical self-assembly $(27)$, will allow fabrication of complex, functional, $2 \mathrm{D}$ and $3 \mathrm{D}$ assemblies that contain multiple components.

\section{References and Notes}

1. M. B. Cohn et al., Proc. SPIE Micromachining and Microfabrication, Santa Clara, CA, 20 to 22 September 1998 (IEEE, New York, 1998), p. 2.

2. R. S. Fearing, IEEE/RSJ, Proc. Int. Intell. Robots Syst. 2 , 212 (1995).

3. H. J. Yeh, J. S. Smith, IEEE Photonics Technol. Lett. 6, 706 (1994).

4. K. Hosokawa, I. Shimoyama, H. Miura, Sens. Actuators A57, 117 (1996).

5. G. W. Dahlman, E. M. Yeatman, Electron. Lett. 36 1707 (2000).

6. U. Srinivasan, D. Liepmann, R. T. Howe, J. MEMS 10 , 17 (2000).

7. R. R. A. Syms, C. Gormley, S. Blackstone, Sens. Actuators A Phys. 88, 273 (2001).

8. E. Smela, O. Ingamas, I. Lundstrom, Science 268, 1735 (1995).

9. K. F. Böhringer, U. Srinivasan, R. T. Howe, IEEE Workshop on Micro Electro Mechanical Systems (MEMS), Interlaken, Switzerland, 21 to 25 January 2001 (IEEE, New York, 2001).

10. G. M. Whitesides, J. P. Mathias, C. T. Seto, Science 254, 1312 (1991).

11. J.-M. Lehn, P. Ball, New Chem. 300 (2000).

12. N. B. Bowden, M. Weck, I. S. Choi, G. M. Whitesides, Acc. Chem. Res. 34, 231 (2001).

13. M. Weck, I. S. Choi, N. L. Jeon, G. M. Whitesides, J. Am. Chem. Soc. 122, 3546 (2000).

14. J. S. Smith, J. Yeh, M. A. Hadley, A. K. Verma, U.S. Patent 5,904,545 (1999).

15. J. S. Smith, J. Yeh, U.S. Patent 5,824,186 (1998)

16. C. D. Fung, P. W. Cheung, W. H. Ko, D. G. Fleming, Eds., Micromachining and Micropackaging of Transducers (Elsevier, Amsterdam, 1985).

17. J. W. Sliwa Jr., U.S. Patent 5,075,253 (1991)

18. D. H. Gracias, J. Tien, T. L. Breen, C. Hsu, G. M. Whitesides, Science 289, 1170 (2000).

19. M. Boncheva, D. H. Gracias, H. O. Jacobs, G. M. Whitesides, Proc. Natl. Acad. Soc. U.S.A., in press.

20. L. F. Miller, IBM J. Res. Dev. 13, 239 (1969).

21. R. R. A. Syms, Sens. Actuators A 65, 238 (1998).

22. F. K. Harsh, V. M. Bright, Y. C. Lee, Sens. Actuators A 77, 237 (1999).

23. We call these patterned drops of solder "receptors" because the interfacial interactions by which they recognize the LEDs are loosely analogous to the hydrophobic interactions that dominate the molecular recognition of ligands by proteins.

24. Additional information about the fabrication of the receptor arrays, the LEDs, and the silicon blocks, as well as procedures to control the surface chemistries, are provided in the supplemental material on Science Online at www.sciencemag.org/cgi/content/full/296/ 5566/323/DC1.

25. N. Bowden, A. Terfort, J. Carbeck, G. M. Whitesides, Science 276, 233 (1997).

26. P. W. Rothemund, Proc. Natl. Acad. Sci. U.S.A. 97, 984 (2000).

27. I. S. Choi, N. Bowden, G. M. Whitesides, Angew. Chem. Int. Ed. Engl. 38, 3078 (1999).

28. We thank J. Kim, Opto Diode Corp. (www. optodiode.com), for providing the LED material. This work was supported by the Swiss National Science Foundation, Deutsche Forschungsgemeinschaft, National Science Foundation (grant CHE-9901358), and Defense Advanced Research Projects Agency/Air Force Research Laboratory/Space and Naval Warfare Systems Center San Diego.

19 December 2001; accepted 1 March 2002 